Structure-Property Relationships of Steel Cylindrical Shells

Ruth G. Hidalgo-Hernandez, Paul G. Allison,

March 2012

Brett A. Williams, Luis A. de Béjar, W. Scott Hart, and Jason Morson 


\section{Structure-Property Relationships of Steel Cylindrical Shells}

Ruth G. Hidalgo-Hernandez, Paul G. Allison, Brett A. Williams, Luis A. de Béjar, W. Scott Hart, and Jason Morson

Geotechnical and Structures Laboratory

U.S. Army Engineer Research and Development Center 3909 Halls Ferry Road

Vicksburg, MS 39180-6199

Final report

Approved for public release; distribution is unlimited.

Prepared for U.S. Army Corps of Engineers

Washington, DC 20314-1000 


\section{Abstract}

Personnel of the U.S. Army Engineer Research and Development Center (ERDC), Geotechnical and Structures Laboratory (GSL), Engineering Systems and Materials Division (ESMD), Concrete and Materials Branch (CMB), Vicksburg, Mississippi, were asked to examine the mechanical properties and microstructure characteristics of a commercially available steel cylindrical shell in its as-manufactured condition. To examine the mechanical properties, uniaxial tensile experiments were performed on ASTM dog-bone specimens and hoop cross-sectional specimens at displacement rates of $0.1 \mathrm{in} . / \mathrm{min}$., to obtain the stress-strain material responses. A commercially available image-analysis program determined the void volume fraction (VVF), the nearest neighbor distance (NND) of pores, maximum and minimum pore sizes, and aspect ratio of the pores at 30-degree increments of the steel hoop cross-sections. The results of the investigation allow for an improved understanding of the mechanical behavior of steel cylindrical shells.

DISCLAIMER: The contents of this report are not to be used for advertising, publication, or promotional purposes. Citation of trade names does not constitute an official endorsement or approval of the use of such commercial products. All product names and trademarks cited are the property of their respective owners. The findings of this report are not to be construed as an official Department of the Army position unless so designated by other authorized documents. 


\section{Contents}

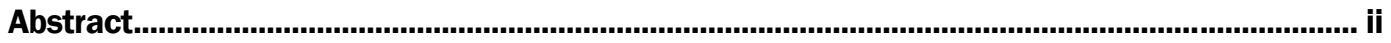

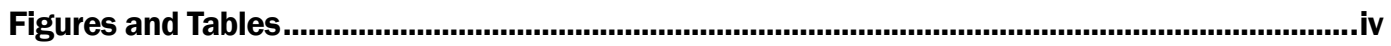

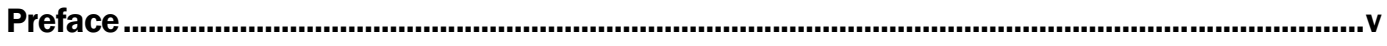

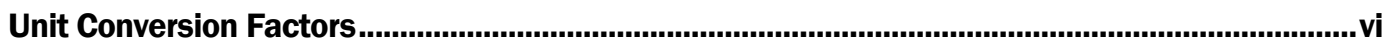

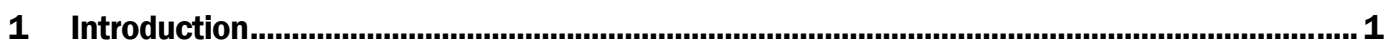

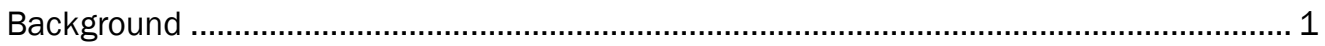

Purpose and scope ............................................................................................... 1

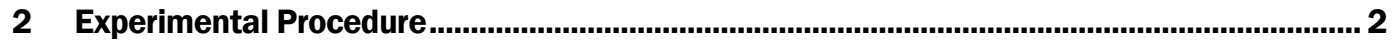

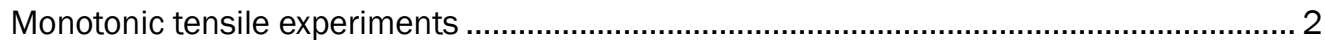

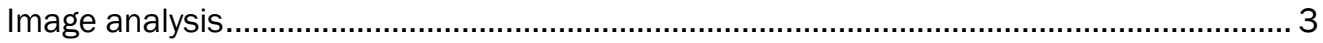

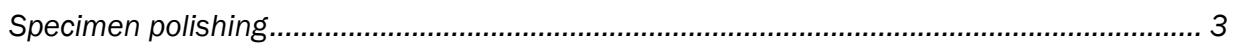

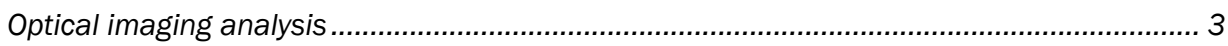

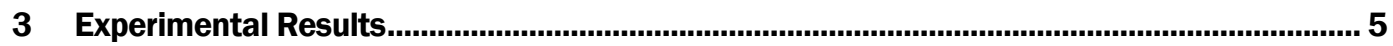

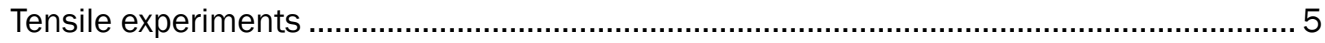

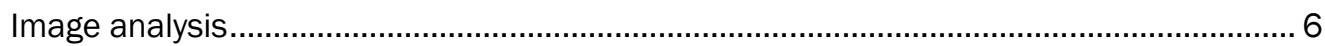

4 Summary

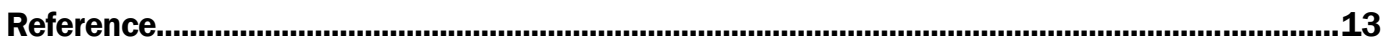

\section{Report Documentation Page}




\section{Figures and Tables}

\section{Figures}

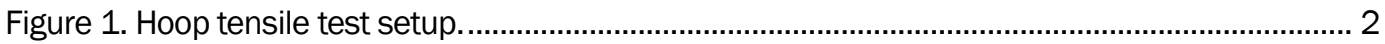

Figure 2. Illustration of the locations of images on hoop cross-sections............................................. 4

Figure 3. Porosity optical micrographs of Specimen 1 at 0-degree location A.................................... 4

Figure 4. Engineering stress versus strain data for the uniaxial dog-bone experiments. ................... 5

Figure 5. Force versus displacement data for hoop tensile testing. ……........................................ 6

\section{Tables}

Table 1. Details of polishing procedure 3

Table 2. Mechanical properties of monotonic uniaxial dog-bone tensile specimens.......................... 5

Table 3. Maximum force and displacement values from hoop tensile experiments.......................... 6

Table 4. Image analysis results of hoop Specimen 1 (0-90 degrees). ............................................... 7

Table 5. Image analysis results of hoop Specimen 1 (120-210 degrees). ....................................... 7

Table 6. Image analysis results of hoop Specimen 1 (240-330 degrees). ...................................... 8

Table 7. Image analysis results of hoop Specimen 2 (0-90 degrees).............................................. 8

Table 8. Image analysis results of hoop Specimen 2 (120-210 degrees). ...................................... 9

Table 9. Image analysis results of hoop Specimen 2 (240-330 degrees). ....................................... 9

Table 10. Image analysis results of hoop Specimen 3 (0-90 degrees) ........................................... 10

Table 11. Image analysis results of hoop Specimen 3 (120-210 degrees) .................................... 10

Table 12. Image analysis results of hoop Specimen 3 (240-330 degrees)................................... 11 


\section{Preface}

This study of steel cylindrical shells was conducted in 2011 by personnel of the U.S. Army Engineer Research and Development Center (ERDC), Geotechnical and Structures Laboratory (GSL), Vicksburg, Mississippi. It was funded through the Military Engineering Basic Research Program, Work Unit ARo31, "Geo-statistical characterization of micro-structural defects and statistical physics modeling of shell-case fragmentation in ammunition steel cases.” The Work Unit Principal Investigator was Dr. Luis A. de Béjar of the GSL's Structural Mechanics Branch (SMB).

The research was conducted by the GSL's Engineering Systems and Materials Division (ESMD), Concrete and Materials Branch (CMB). Laboratory characterization tests and analyses of the results were performed by Ruth G. Hidalgo-Hernandez, Dr. Paul A. Allison, Brett A. Williams, W. Scott Hart, and Jason Morson, all of the CMB. HidalgoHernandez and Allison prepared this report.

During the investigation, Christopher M. Moore was Acting Chief, CMB; Dr. Larry N. Lynch was Chief, ESMD; Dr. William P. Grogan was Deputy Director, GSL; and Dr. David W. Pittman was Director, GSL.

COL Kevin J. Wilson was Commander and Executive Director of ERDC. Dr. Jeffery P. Holland was Director. 


\section{Unit Conversion Factors}

\begin{tabular}{|l|c|l|}
\hline Multiply & By & To Obtain \\
\hline degrees (angle) & 0.01745329 & radians \\
\hline feet & 0.3048 & meters \\
\hline foot-pounds force & 1.355818 & joules \\
\hline inches & 0.0254 & meters \\
\hline inch-pounds (force) & 0.1129848 & newton meters \\
\hline microns & $1.0 \mathrm{E}-06$ & meters \\
\hline pounds (force) & 4.448222 & newtons \\
\hline pounds (force) per foot & 14.59390 & newtons per meter \\
\hline pounds (force) per inch & 175.1268 & newtons per meter \\
\hline pounds (force) per square foot & 47.88026 & pascals \\
\hline pounds (force) per square inch & 6.894757 & kilopascals \\
\hline
\end{tabular}




\section{Introduction}

\section{Background}

Personnel of the U.S. Army Engineer Research and Development Center (ERDC), Geotechnical and Structures Laboratory (GSL), conducted a series of laboratory experiments to measure the mechanical properties and evaluate the microstructure characteristics of a commercially available steel pipe in its as-manufactured condition. The mechanical property tests consisted of uniaxial tensile experiments performed on dog-bone specimens and hoop cross-sectional specimens at a constant displacement rate. In addition, a commercially available image-analysis program was used to determine the microstructural features such as void volume fraction, maximum and minimum pore size, the distance to the pores' nearest neighbors, and aspect ratio of the pores at 30-degree increments of the steel hoop cross-sections.

\section{Purpose and scope}

The purpose of this project was to obtain baseline experimental data on mechanical behavior and microstructure of the steel cylindrical shells. The collected data will be used for the assessment of geo-statistical techniques that simulate cross-sectional porosity for mechanical analyses of crack initiation, growth, propagation, and coalescence when subjected to impulsive internal loads.

Chapter 2 provides a description of the experimental procedures, and experimental results are in Chapter 3. A summary of the investigation is in Chapter 4. 


\section{Experimental Procedure}

\section{Monotonic tensile experiments}

An MTS 110-kip load frame was used to conduct three monotonic tensile experiments on a low-carbon-alloy steel pipe. Experiments were performed on uniaxial dog-bone-shaped tensile specimens in the longitudinal orientation of the pipe and hoop tensile specimens in the pipe's transverse orientation. The ERDC machine shop prepared the test specimens using a water-jet cutting system.

For the uniaxial monotonic tensile experiments, the load frame monitored force, while an MTS 634.12E-24 extensometer with a 1-in. gage length provided strain data. The experiments were executed at a displacement control rate of $0.1 \mathrm{in} . / \mathrm{min}$.

During hoop tensile testing, the load frame monitored force and displacement. Strain data were not available. The hoop tests also were performed in displacement control at a rate of $0.1 \mathrm{in} . / \mathrm{min}$. The experimental setup is shown in Figure 1.

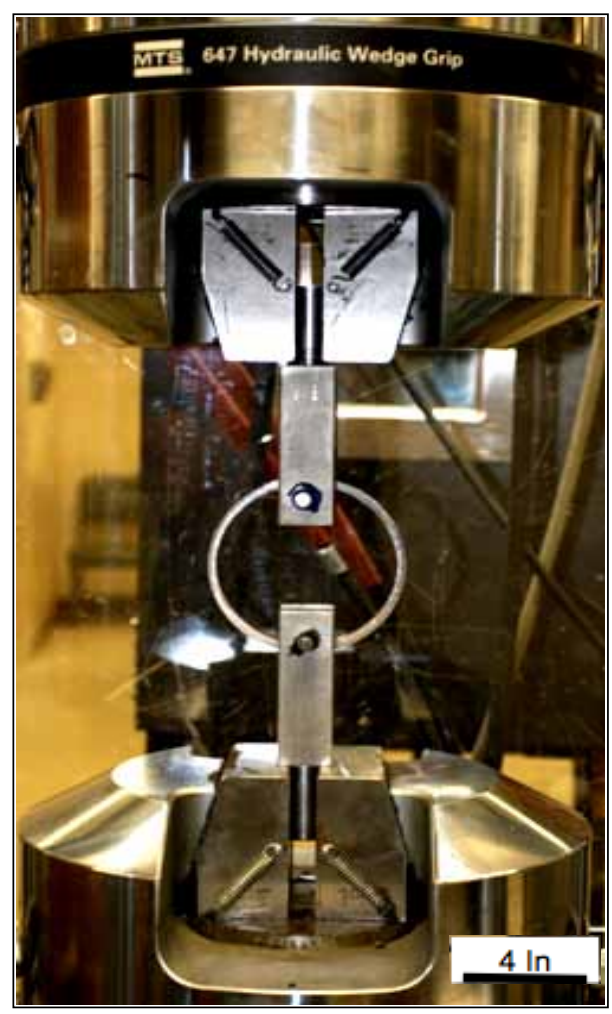

Figure 1. Hoop tensile test setup. 


\section{Image analysis}

\section{Specimen polishing}

Sectioned hoop specimens were hand-polished on an Allied High Tech MultiPrep polishing system. The specimens were polished to a surface deviation of $+/-1 \mu \mathrm{m}$ using the procedure outlined in Table 1 . Between each polishing step, sonication using distilled water cleaned the specimens.

Table 1. Details of polishing procedure.

\begin{tabular}{|l|l|l|l|}
\hline & Abrasive & Lubricant & Time (min) \\
\hline Step 1 & 60-grit silicon carbide & distilled water & until plane \\
\hline Step 2 & 120-grit silicon carbide & distilled water & $3: 00$ \\
\hline Step 3 & 240-grit silicon carbide & distilled water & $3: 00$ \\
\hline Step 4 & 400-grit silicon carbide & distilled water & $3: 00$ \\
\hline Step 5 & 600-grit silicon carbide & distilled water & $3: 00$ \\
\hline Step 6 & 800-grit silicon carbide & distilled water & $3: 00$ \\
\hline Step 7 & 1200-grit silicon carbide & distilled water & $3: 00$ \\
\hline Step 8 & 1 um diamond paste & diamond extender & $3: 00$ \\
\hline
\end{tabular}

\section{Optical imaging analysis}

A Zeiss Z9Imager captured darkfield images of the polished hoop crosssections at 30-degree increments. Images were obtained at three locations for each increment around the hoop specimen, as illustrated in Figure 2. The first image was recorded halfway between the inner edge of the specimen and the midpoint. The second image was captured at the midpoint of the specimen, and the third image was obtained halfway between the midpoint and the outer edge of the specimen. A typical image is shown in Figure 3.

An image-analysis software program developed by the Center for Advanced Vehicular Systems at Mississippi State University (MSU-CAVS) calculated void volume fraction (VVF), nearest neighbor distance (NND), pore aspect ratio, and maximum and minimum pore size from each image. T.Y. Stone and others detail the image-analysis procedure used for specimens in the research in their 2009 article in the Journal of Powder Metallurgy. 


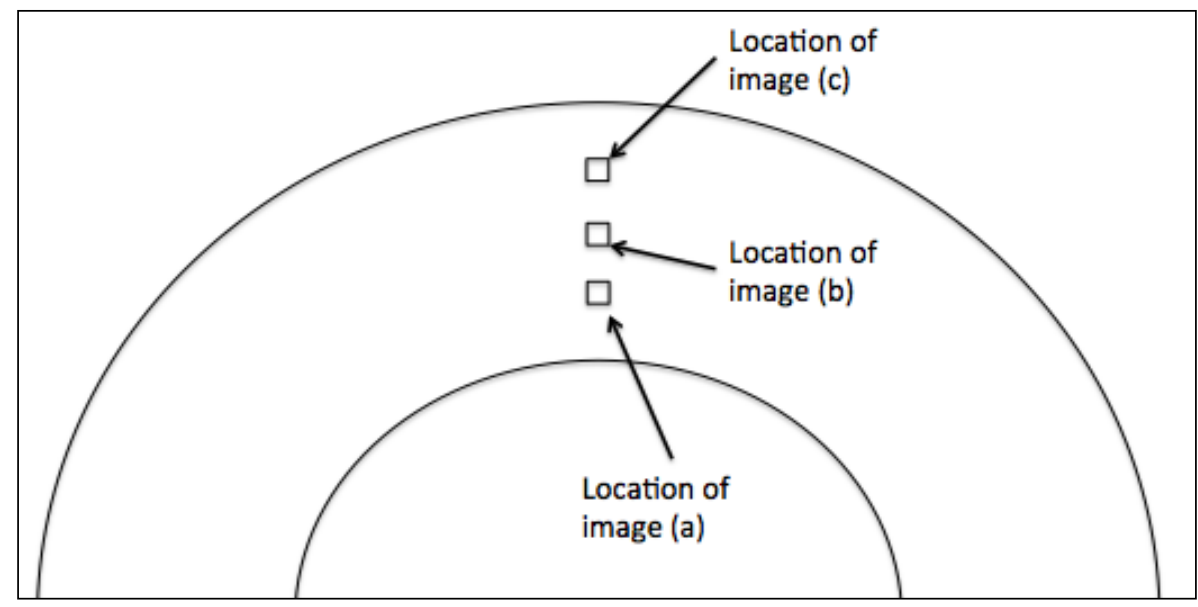

Figure 2. Illustration of the locations of images on hoop cross-sections.

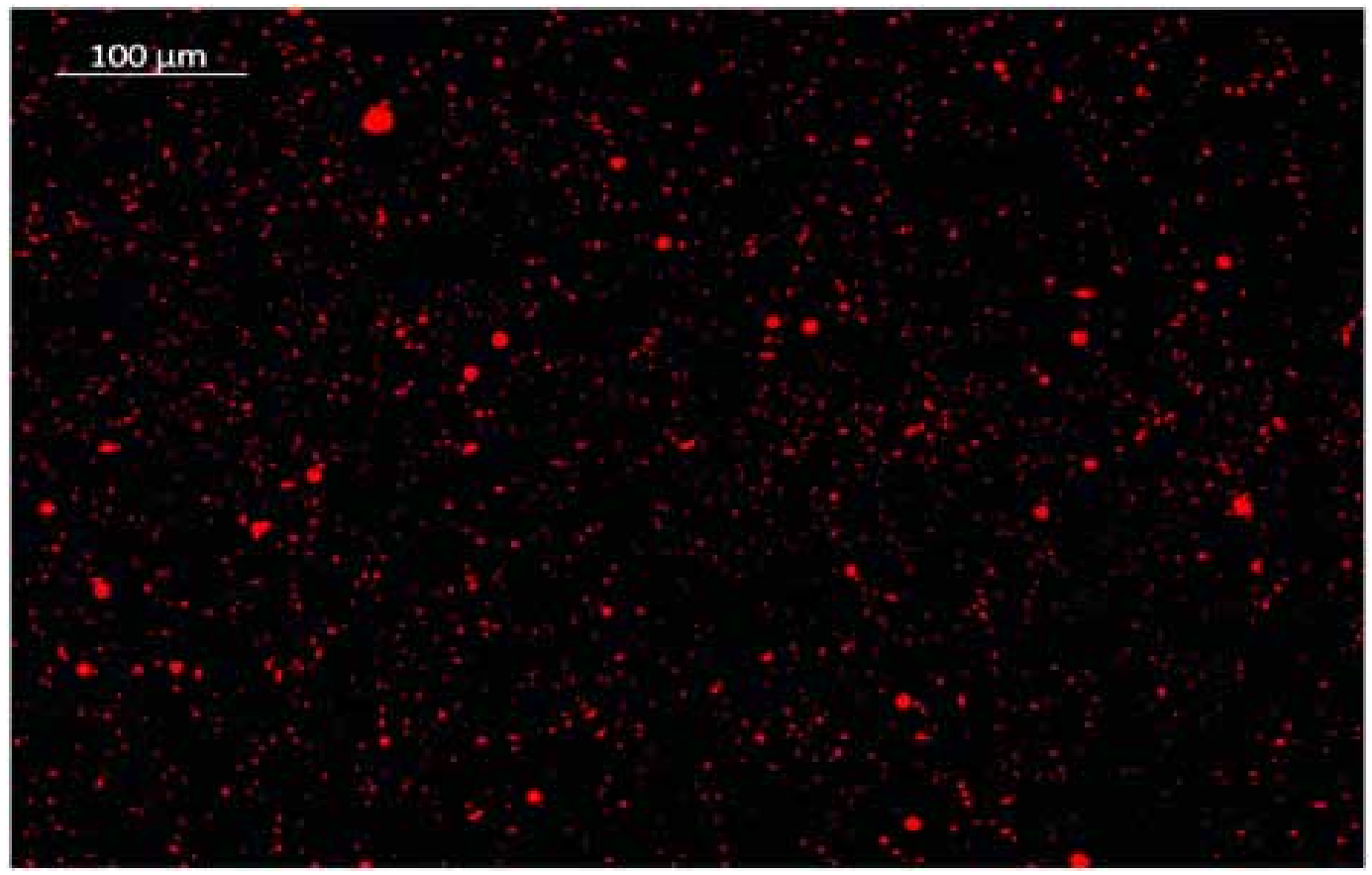

Figure 3. Porosity optical micrographs of Specimen 1 at 0-degree location A. 


\section{Experimental Results}

\section{Tensile experiments}

The results of the uniaxial dog-bone tensile experiments are in Figure 4.

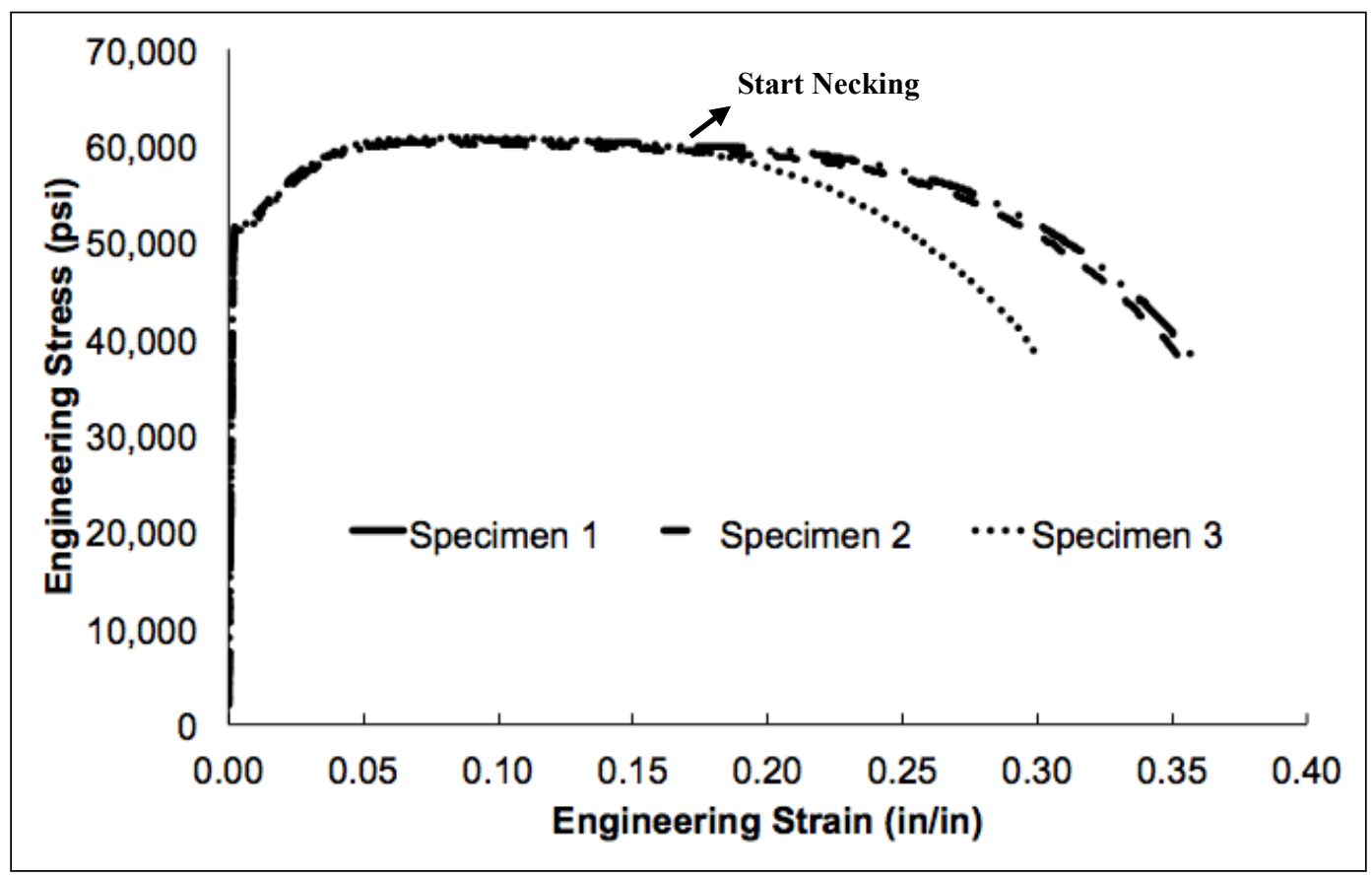

Figure 4. Engineering stress versus strain data for the uniaxial dog-bone experiments.

All three specimens in Figure 4 exhibit similar hardening and recovery in the stress-strain responses. However, the strains to failure for the specimens vary from 0.30 to $0.36 \mathrm{in}$./in. Young's modulus, ultimate tensile strength, and strain to failure from these experiments are in Table 2.

The force versus displacement relations for the three hoop specimens are in Figure 5 .

Table 2. Mechanical properties of monotonic uniaxial dog-bone tensile specimens.

\begin{tabular}{|l|l|l|l|l|}
\hline & $\begin{array}{l}\text { Ultimate Tensile } \\
\text { Strength } \\
\text { (ksi) }\end{array}$ & Young's Modulus (ksi) & $\begin{array}{l}\text { Strain-to-failure } \\
\text { (in/in) }\end{array}$ & $\begin{array}{l}\text { Yield Stress } \\
\text { (ksi) }\end{array}$ \\
\hline Specimen 1 & 61.1 & 27.1 & 0.36 & 51.1 \\
\hline Specimen 2 & 60.4 & 27.5 & 0.35 & 51.1 \\
\hline Specimen 3 & 61.0 & 28.3 & 0.30 & 51.2 \\
\hline
\end{tabular}




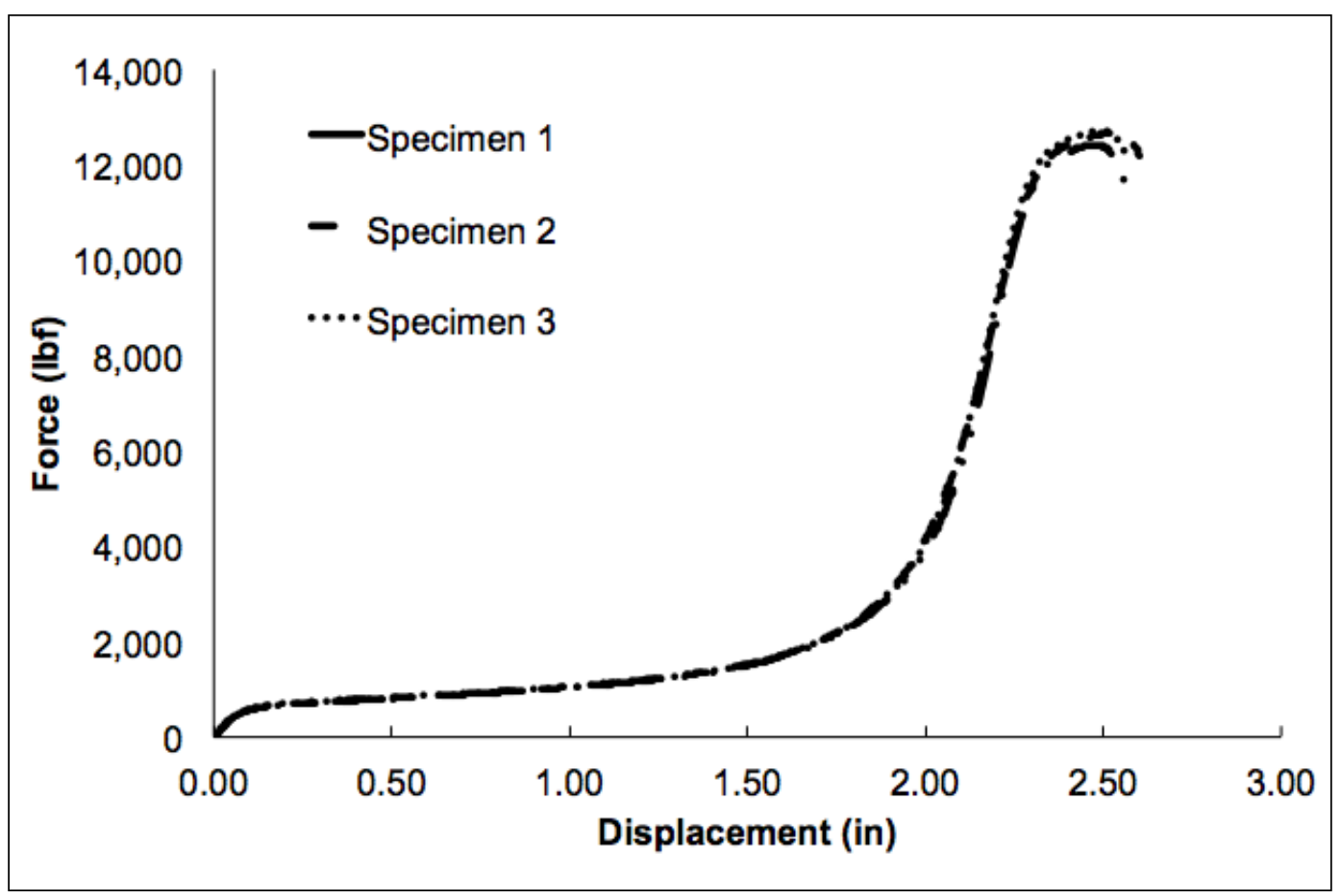

Figure 5. Force versus displacement data for hoop tensile testing.

The data appear similar. The values of maximum force and maximum displacement are in Table 3.

Table 3. Maximum force and displacement values from hoop tensile experiments.

\begin{tabular}{|l|l|l|}
\hline & $\begin{array}{l}\text { Maximum Force } \\
\text { (lbf) }\end{array}$ & $\begin{array}{l}\text { Maximum Displacement } \\
\text { (in) }\end{array}$ \\
\hline Specimen 1 & 12418 & 2.56003 \\
\hline Specimen 2 & 12666 & 2.60156 \\
\hline Specimen 3 & 12706 & 2.56348 \\
\hline
\end{tabular}

\section{Image analysis}

The image analysis results featuring VVF, maximum and minimum pore size, NND, and aspect ratio of the pores in the hoop cross-sections are in Tables 4 through 12. The images were taken at a magnification of $20 \mathrm{X}$. The minimum pore area that could be resolved at the selected magnification was $1.16 \mu \mathrm{m}$. 
Table 4. Image analysis results of hoop Specimen 1 (0-90 degrees).

\begin{tabular}{|c|c|c|c|c|c|c|c|c|c|c|}
\hline & \multirow{2}{*}{$\begin{array}{l}\text { Void } \\
\text { Volume } \\
\text { Fraction }\end{array}$} & \multicolumn{3}{|c|}{ Pore Area } & \multicolumn{3}{|c|}{$\begin{array}{l}\text { Nearest Neighbor } \\
\text { Distance }\end{array}$} & \multicolumn{3}{|c|}{ Aspect Ratio } \\
\hline & & $\begin{array}{l}\text { Max. } \\
(\mu \mathrm{m})\end{array}$ & $\begin{array}{l}\text { Min. } \\
(\mu \mathrm{m})\end{array}$ & $\begin{array}{l}\text { Avg. } \\
(\mu \mathrm{m})\end{array}$ & $\begin{array}{l}\text { Max. } \\
(\mu \mathrm{m})\end{array}$ & $\begin{array}{l}\text { Min. } \\
(\mu \mathrm{m})\end{array}$ & $\begin{array}{l}\text { Avg. } \\
(\mu \mathrm{m})\end{array}$ & $\begin{array}{l}\text { Max. } \\
(\mu \mathrm{m})\end{array}$ & $\begin{array}{l}\text { Min. } \\
(\mu \mathrm{m})\end{array}$ & $\begin{array}{l}\text { Avg. } \\
(\mu \mathrm{m})\end{array}$ \\
\hline Oa & 0.0282 & 217 & 1.16 & 4.60 & 25.48 & 1.84 & 6.80 & 4.56 & 1.00 & 1.42 \\
\hline $\mathrm{Ob}$ & 0.0437 & 1408 & 1.16 & 6.89 & 22.11 & 1.70 & 6.72 & 4.24 & 1.00 & 1.41 \\
\hline Oc & 0.0427 & 4002 & 1.16 & 6.73 & 30.16 & 1.61 & 6.83 & 4.88 & 1.00 & 1.39 \\
\hline $30 a$ & 0.0039 & 128 & 1.16 & 4.87 & 102.16 & 1.78 & 17.64 & 4.56 & 1.00 & 1.45 \\
\hline $30 \mathrm{~b}$ & 0.0035 & 52 & 1.16 & 5.53 & 91.78 & 1.81 & 18.95 & 3.62 & 1.00 & 1.44 \\
\hline $30 c$ & 0.0034 & 60 & 1.16 & 6.51 & 98.29 & 2.32 & 21.84 & 3.13 & 1.00 & 1.39 \\
\hline $60 a$ & 0.0177 & 91 & 1.16 & 3.98 & 34.92 & 1.75 & 7.72 & 4.36 & 1.00 & 1.54 \\
\hline $60 \mathrm{~b}$ & 0.0225 & 1580 & 1.16 & 5.34 & 43.68 & 1.31 & 7.60 & 4.83 & 1.00 & 1.56 \\
\hline $60 c$ & 0.0080 & 532 & 1.16 & 5.65 & 67.15 & 2.36 & 13.40 & 4.85 & 1.00 & 1.47 \\
\hline $90 a$ & 0.0120 & 507 & 1.16 & 4.21 & 41.89 & 1.62 & 9.34 & 3.49 & 1.00 & 1.45 \\
\hline $90 b$ & 0.0120 & 323 & 1.16 & 6.79 & 39.05 & 2.03 & 12.37 & 3.67 & 1.00 & 1.42 \\
\hline $90 c$ & 0.0101 & 433 & 1.16 & 6.17 & 50.78 & 1.72 & 12.50 & 5.75 & 1.00 & 1.40 \\
\hline
\end{tabular}

Table 5. Image analysis results of hoop Specimen 1 (120-210 degrees).

\begin{tabular}{|c|c|c|c|c|c|c|c|c|c|c|}
\hline & \multirow{2}{*}{$\begin{array}{l}\text { Void } \\
\text { Volume } \\
\text { Fraction }\end{array}$} & \multicolumn{3}{|c|}{ Pore Area } & \multicolumn{3}{|c|}{$\begin{array}{l}\text { Nearest Neighbor } \\
\text { Distance }\end{array}$} & \multicolumn{3}{|c|}{ Aspect Ratio } \\
\hline & & $\begin{array}{l}\text { Max. } \\
(\mu \mathrm{m})\end{array}$ & $\begin{array}{l}\text { Min. } \\
(\mu \mathrm{m})\end{array}$ & $\begin{array}{l}\text { Avg. } \\
(\mu \mathrm{m})\end{array}$ & $\begin{array}{l}\text { Max. } \\
(\mu \mathrm{m})\end{array}$ & $\begin{array}{l}\text { Min. } \\
(\mu \mathrm{m})\end{array}$ & $\begin{array}{l}\text { Avg. } \\
(\mu \mathrm{m})\end{array}$ & $\begin{array}{l}\text { Max. } \\
(\mu \mathrm{m})\end{array}$ & $\begin{array}{l}\text { Min. } \\
(\mu \mathrm{m})\end{array}$ & $\begin{array}{l}\text { Avg. } \\
(\mu \mathrm{m})\end{array}$ \\
\hline $120 a$ & 0.0086 & 116 & 1.16 & 4.63 & 47.63 & 1.96 & 12.02 & 3.49 & 1.00 & 1.43 \\
\hline $120 \mathrm{~b}$ & 0.0194 & 1261 & 1.16 & 10.10 & 55.68 & 1.84 & 10.29 & 4.58 & 1.00 & 1.49 \\
\hline $120 c$ & 0.0174 & 1226 & 1.16 & 15.00 & 58.18 & 1.86 & 14.55 & 4.12 & 1.00 & 1.53 \\
\hline $150 a$ & 0.0151 & 448 & 1.16 & 4.78 & 27.21 & 1.64 & 9.01 & 5.96 & 1.00 & 1.39 \\
\hline $150 b$ & 0.0096 & 565 & 1.16 & 6.35 & 53.99 & 2.27 & 12.99 & 4.59 & 1.00 & 1.41 \\
\hline $150 c$ & 0.0143 & 292 & 1.16 & 4.95 & 30.20 & 1.87 & 9.86 & 3.94 & 1.00 & 1.37 \\
\hline $180 a$ & 0.0153 & 107 & 1.16 & 4.17 & 32.71 & 1.87 & 8.72 & 3.79 & 1.00 & 1.38 \\
\hline $180 \mathrm{~b}$ & 0.0157 & 73 & 1.16 & 4.74 & 45.64 & 1.80 & 8.81 & 4.00 & 1.00 & 1.43 \\
\hline $180 c$ & 0.0230 & 3310 & 1.16 & 13.48 & 55.66 & 1.75 & 10.33 & 5.54 & 1.00 & 1.52 \\
\hline $210 a$ & 0.0094 & 588 & 1.16 & 5.04 & 43.17 & 2.00 & 11.96 & 4.77 & 1.00 & 1.42 \\
\hline $210 b$ & 0.0079 & 177 & 1.16 & 4.12 & 37.64 & 1.62 & 11.34 & 4.02 & 1.00 & 1.47 \\
\hline $210 c$ & 0.0134 & 475 & 1.16 & 4.30 & 27.19 & 1.57 & 8.72 & 5.25 & 1.00 & 1.52 \\
\hline
\end{tabular}


Table 6. Image analysis results of hoop Specimen 1 (240-330 degrees).

\begin{tabular}{|c|c|c|c|c|c|c|c|c|c|c|}
\hline & \multirow{2}{*}{$\begin{array}{l}\text { Void } \\
\text { Volume } \\
\text { Fraction }\end{array}$} & \multicolumn{3}{|c|}{ Pore Area } & \multicolumn{3}{|c|}{$\begin{array}{l}\text { Nearest Neighbor } \\
\text { Distance }\end{array}$} & \multicolumn{3}{|c|}{ Aspect Ratio } \\
\hline & & $\begin{array}{l}\text { Max. } \\
(\mu \mathrm{m})\end{array}$ & $\begin{array}{l}\text { Min. } \\
(\mu \mathrm{m})\end{array}$ & $\begin{array}{l}\text { Avg. } \\
(\mu \mathrm{m})\end{array}$ & $\begin{array}{l}\text { Max. } \\
(\mu \mathrm{m})\end{array}$ & $\begin{array}{l}\text { Min. } \\
(\mu \mathrm{m})\end{array}$ & $\begin{array}{l}\text { Avg. } \\
(\mu \mathrm{m})\end{array}$ & $\begin{array}{l}\text { Max. } \\
(\mu \mathrm{m})\end{array}$ & $\begin{array}{l}\text { Min. } \\
(\mu \mathrm{m})\end{array}$ & $\begin{array}{l}\text { Avg. } \\
(\mu \mathrm{m})\end{array}$ \\
\hline $240 a$ & 0.0102 & 294 & 1.16 & 3.86 & 51.32 & 1.83 & 9.71 & 5.00 & 1.00 & 1.48 \\
\hline $240 b$ & 0.0050 & 151 & 1.16 & 3.29 & 42.14 & 2.01 & 12.41 & 7.59 & 1.00 & 1.51 \\
\hline $240 c$ & 0.0131 & 612 & 1.16 & 3.66 & 35.87 & 1.61 & 8.17 & 4.47 & 1.00 & 1.46 \\
\hline $270 a$ & 0.0024 & 47 & 1.16 & 3.90 & 66.34 & 1.89 & 19.61 & 3.27 & 1.00 & 1.42 \\
\hline $270 b$ & 0.0057 & 78 & 1.16 & 2.87 & 52.44 & 1.64 & 11.04 & 4.00 & 1.00 & 1.46 \\
\hline $270 c$ & 0.0050 & 272 & 1.16 & 4.24 & 64.97 & 1.78 & 13.56 & 5.99 & 1.00 & 1.47 \\
\hline $300 a$ & 0.0046 & 67 & 1.16 & 3.66 & 61.44 & 2.37 & 13.29 & 7.72 & 1.00 & 1.48 \\
\hline $300 \mathrm{~b}$ & 0.0049 & 1294 & 1.16 & 16.87 & 78.27 & 2.49 & 25.03 & 3.27 & 1.00 & 1.36 \\
\hline $300 c$ & 0.0039 & 104 & 1.16 & 4.08 & 98.84 & 2.01 & 14.24 & 3.50 & 1.00 & 1.44 \\
\hline $330 a$ & 0.0042 & 383 & 1.16 & 9.63 & 69.75 & 3.29 & 27.15 & 3.18 & 1.00 & 1.42 \\
\hline $330 \mathrm{~b}$ & 0.0035 & 37 & 1.16 & 3.55 & 47.75 & 2.04 & 15.31 & 4.08 & 1.00 & 1.46 \\
\hline $330 c$ & 0.0032 & 40 & 1.16 & 4.20 & 64.25 & 1.71 & 18.37 & 3.85 & 1.00 & 1.42 \\
\hline
\end{tabular}

Table 7. Image analysis results of hoop Specimen 2 (0-90 degrees).

\begin{tabular}{|c|c|c|c|c|c|c|c|c|c|c|}
\hline & \multirow{2}{*}{\begin{tabular}{|l} 
Void \\
Volume \\
Fraction
\end{tabular}} & \multicolumn{3}{|c|}{ Pore Area } & \multicolumn{3}{|c|}{$\begin{array}{l}\text { Nearest Neighbor } \\
\text { Distance }\end{array}$} & \multicolumn{3}{|c|}{ Aspect Ratio } \\
\hline & & $\begin{array}{l}\text { Max. } \\
(\mu \mathrm{m})\end{array}$ & $\begin{array}{l}\text { Min. } \\
(\mu \mathrm{m})\end{array}$ & $\begin{array}{l}\text { Avg. } \\
(\mu \mathrm{m})\end{array}$ & $\begin{array}{l}\text { Max. } \\
(\mu \mathrm{m})\end{array}$ & $\begin{array}{l}\text { Min. } \\
(\mu \mathrm{m})\end{array}$ & $\begin{array}{l}\text { Avg. } \\
(\mu \mathrm{m})\end{array}$ & $\begin{array}{l}\text { Max. } \\
(\mu \mathrm{m})\end{array}$ & $\begin{array}{l}\text { Min. } \\
(\mu \mathrm{m})\end{array}$ & $\begin{array}{l}\text { Avg. } \\
(\mu \mathrm{m})\end{array}$ \\
\hline $0 a$ & 0.0211 & 621.17 & 1.16 & 5.7 & 31.63 & 1.57 & 8.58 & 6 & 1 & 1.58 \\
\hline $\mathrm{Ob}$ & 0.0325 & 333.56 & 1.16 & 7.91 & 28.95 & 1.81 & 7.88 & 4.12 & 1 & 1.6 \\
\hline Oc & 0.0320 & 366.52 & 1.16 & 8.11 & 31.95 & 1.79 & 7.92 & 4.45 & 1 & 1.58 \\
\hline $30 a$ & 0.0099 & 361.02 & 1.16 & 6.14 & 63.41 & 1.83 & 11.52 & 5.05 & 1 & 1.53 \\
\hline $30 \mathrm{~b}$ & 0.0133 & 247.14 & 1.16 & 7.48 & 59.7 & 1.87 & 10.44 & 5.05 & 1 & 1.62 \\
\hline $30 c$ & 0.0055 & 42.78 & 1.16 & 4.05 & 63.87 & 1.94 & 11.78 & 4.47 & 1 & 1.58 \\
\hline $60 a$ & 0.0026 & 128.92 & 1.16 & 3.56 & 67 & 1.83 & 17.23 & 3.85 & 1 & 1.55 \\
\hline $60 \mathrm{~b}$ & 0.0060 & 67.64 & 1.16 & 2.47 & 58.3 & 1.7 & 10.03 & 5 & 1 & 1.47 \\
\hline $60 c$ & 0.0339 & 244.25 & 1.15 & 3.2 & 24.82 & 1.53 & 5.02 & 5.24 & 1 & 1.57 \\
\hline $90 a$ & 0.0035 & 44.22 & 1.16 & 2.39 & 52.61 & 1.94 & 11.84 & 3.53 & 1 & 1.44 \\
\hline $90 \mathrm{~b}$ & 0.0017 & 41.05 & 1.16 & 2.86 & 93.64 & 2.03 & 17.92 & 3.09 & 1 & 1.49 \\
\hline $90 c$ & 0.0217 & 239.05 & 1.16 & 4.19 & 39.96 & 1.48 & 6.31 & 5 & 1 & 1.64 \\
\hline
\end{tabular}


Table 8. Image analysis results of hoop Specimen 2 (120-210 degrees).

\begin{tabular}{|c|c|c|c|c|c|c|c|c|c|c|}
\hline & \multirow{2}{*}{$\begin{array}{l}\text { Void } \\
\text { Volume } \\
\text { Fraction }\end{array}$} & \multicolumn{3}{|c|}{ Pore Area } & \multicolumn{3}{|c|}{$\begin{array}{l}\text { Nearest Neighbor } \\
\text { Distance }\end{array}$} & \multicolumn{3}{|c|}{ Aspect Ratio } \\
\hline & & $\begin{array}{l}\text { Max. } \\
(\mu \mathrm{m})\end{array}$ & $\begin{array}{l}\text { Min. } \\
(\mu \mathrm{m})\end{array}$ & $\begin{array}{l}\text { Avg. } \\
(\mu \mathrm{m})\end{array}$ & $\begin{array}{l}\text { Max. } \\
(\mu \mathrm{m})\end{array}$ & $\begin{array}{l}\text { Min. } \\
(\mu \mathrm{m})\end{array}$ & $\begin{array}{l}\text { Avg. } \\
(\mu \mathrm{m})\end{array}$ & $\begin{array}{l}\text { Max. } \\
(\mu \mathrm{m})\end{array}$ & $\begin{array}{l}\text { Min. } \\
(\mu \mathrm{m})\end{array}$ & $\begin{array}{l}\text { Avg. } \\
(\mu \mathrm{m})\end{array}$ \\
\hline $120 a$ & 0.0204 & 128.63 & 1.16 & 7.88 & 40.53 & 2.05 & 9.12 & 6.00 & 1.00 & 1.67 \\
\hline $120 b$ & 0.0286 & 248.58 & 1.16 & 7.55 & 34.95 & 0.08 & 7.55 & 8.15 & 1.00 & 1.6 \\
\hline $120 c$ & 0.0643 & 559.02 & 1.16 & 5.92 & 31.29 & 1.149 & 4.74 & 6.15 & 1.00 & 1.67 \\
\hline $150 a$ & 0.0134 & 130 & 1.16 & 6.36 & 49.41 & 1.79 & 9.73 & 7.92 & 1.00 & 1.65 \\
\hline $150 b$ & 0.0083 & 349 & 1.16 & 6.31 & 54.22 & 1.86 & 10.57 & 6.26 & 1.00 & 1.64 \\
\hline $150 c$ & 0.0145 & 210 & 1.16 & 7.38 & 38.64 & 1.64 & 9.86 & 7.64 & 1.00 & 1.67 \\
\hline $180 a$ & 0.0355 & 337 & 1.16 & 6.91 & 29.20 & 1.63 & 7.21 & 5.65 & 1.00 & 1.56 \\
\hline $180 \mathrm{~b}$ & 0.0299 & 148 & 1.16 & 6.46 & 28.40 & 1.61 & 7.38 & 5.47 & 1.00 & 1.59 \\
\hline $180 c$ & 0.0245 & 165 & 1.16 & 4.35 & 28.32 & 1.57 & 6.74 & 10.00 & 1.00 & 1.55 \\
\hline $210 a$ & 0.0194 & 103 & 1.16 & 6.57 & 36.11 & 1.61 & 8.57 & 5.84 & 1.00 & 1.67 \\
\hline $210 b$ & 0.0372 & 398 & 1.16 & 9.25 & 28.66 & 1.61 & 7.72 & 7.85 & 1.00 & 1.63 \\
\hline $210 c$ & 0.0300 & 188 & 1.16 & 5.82 & 28.83 & 1.88 & 7.08 & 7.43 & 1.00 & 1.57 \\
\hline
\end{tabular}

Table 9. Image analysis results of hoop Specimen 2 (240-330 degrees).

\begin{tabular}{|c|c|c|c|c|c|c|c|c|c|c|}
\hline & \multirow{2}{*}{$\begin{array}{l}\text { Void } \\
\text { Volume } \\
\text { Fraction }\end{array}$} & \multicolumn{3}{|c|}{ Pore Area } & \multicolumn{3}{|c|}{$\begin{array}{l}\text { Nearest Neighbor } \\
\text { Distance }\end{array}$} & \multicolumn{3}{|c|}{ Aspect Ratio } \\
\hline & & $\begin{array}{l}\text { Max. } \\
(\mu \mathrm{m})\end{array}$ & $\begin{array}{l}\text { Min. } \\
(\mu \mathrm{m})\end{array}$ & $\begin{array}{l}\text { Avg. } \\
(\mu \mathrm{m})\end{array}$ & $\begin{array}{l}\text { Max. } \\
(\mu \mathrm{m})\end{array}$ & $\begin{array}{l}\text { Min. } \\
(\mu \mathrm{m})\end{array}$ & $\begin{array}{l}\text { Avg. } \\
(\mu \mathrm{m})\end{array}$ & $\begin{array}{c}\text { Max. } \\
(\mu \mathrm{m})\end{array}$ & $\begin{array}{l}\text { Min. } \\
(\mu \mathrm{m})\end{array}$ & $\begin{array}{l}\text { Avg. } \\
(\mu \mathrm{m})\end{array}$ \\
\hline $240 a$ & 0.0147 & 82 & 1.16 & 3.87 & 30.96 & 1.64 & 8.06 & 6.18 & 1.00 & 1.53 \\
\hline $240 b$ & 0.0219 & 251 & 1.16 & 4.49 & 31.72 & 1.67 & 7.19 & 4.55 & 1.00 & 1.56 \\
\hline $240 c$ & 0.0347 & 563 & 1.16 & 3.87 & 35.17 & 1.50 & 5.32 & 4.80 & 1.00 & 1.57 \\
\hline $270 a$ & 0.0340 & 439 & 1.16 & 4.43 & 24.58 & 1.80 & 6.00 & 4.00 & 1.00 & 1.48 \\
\hline $270 b$ & 0.0799 & 344 & 1.16 & 6.63 & 26.43 & 1.70 & 5.13 & 4.98 & 1.00 & 1.50 \\
\hline $270 c$ & 0.0463 & 296 & 1.16 & 7.01 & 30.23 & 1.64 & 6.41 & 5.73 & 1.00 & 1.50 \\
\hline $300 a$ & 0.0524 & 1234 & 1.16 & 5.05 & 25.78 & 1.60 & 5.37 & 7.00 & 1.00 & 1.51 \\
\hline $300 b$ & 0.0392 & 319 & 1.16 & 4.17 & 20.91 & 1.64 & 5.51 & 6.31 & 1.00 & 1.46 \\
\hline $300 c$ & 0.0457 & 746 & 1.16 & 5.27 & 35.28 & 1.64 & 5.88 & 4.52 & 1.00 & 1.47 \\
\hline $330 a$ & 0.0187 & 93 & 1.16 & 3.28 & 32.76 & 1.84 & 6.83 & 6.94 & 1.00 & 1.48 \\
\hline $330 b$ & 0.0419 & 181 & 1.16 & 3.98 & 23.02 & 1.61 & 5.36 & 8.04 & 1.00 & 1.53 \\
\hline $330 c$ & 0.0439 & 237 & 1.16 & 4.98 & 27.36 & 1.61 & 5.66 & 5.34 & 1.00 & 1.50 \\
\hline
\end{tabular}


Table 10. Image analysis results of hoop Specimen 3 (0-90 degrees).

\begin{tabular}{|c|c|c|c|c|c|c|c|c|c|c|}
\hline & \multirow{2}{*}{$\begin{array}{l}\text { Void } \\
\text { Volume } \\
\text { Fraction } \\
\end{array}$} & \multicolumn{3}{|c|}{ Pore Area } & \multicolumn{3}{|c|}{$\begin{array}{l}\text { Nearest Neighbor } \\
\text { Distance }\end{array}$} & \multicolumn{3}{|c|}{ Aspect Ratio } \\
\hline & & $\begin{array}{l}\text { Max. } \\
(\mu \mathrm{m})\end{array}$ & $\begin{array}{l}\text { Min. } \\
(\mu \mathrm{m})\end{array}$ & $\begin{array}{l}\text { Avg. } \\
(\mu \mathrm{m})\end{array}$ & $\begin{array}{l}\text { Max. } \\
(\mu \mathrm{m})\end{array}$ & $\begin{array}{l}\text { Min. } \\
(\mu \mathrm{m})\end{array}$ & $\begin{array}{l}\text { Avg. } \\
(\mu \mathrm{m})\end{array}$ & $\begin{array}{l}\text { Max. } \\
(\mu \mathrm{m})\end{array}$ & $\begin{array}{l}\text { Min. } \\
(\mu \mathrm{m})\end{array}$ & $\begin{array}{l}\text { Avg. } \\
(\mu \mathrm{m})\end{array}$ \\
\hline $0 a$ & 0.0036 & 86.14 & 1.16 & 7.51 & 75.37 & 3.91 & 23.77 & 2.78 & 1.00 & 1.33 \\
\hline $\mathrm{Ob}$ & 0.0042 & 211.59 & 1.16 & 8.91 & 71.07 & 2.92 & 24.40 & 2.86 & 1.00 & 1.32 \\
\hline Oc & 0.0042 & 82.96 & 1.16 & 10.70 & 77.76 & 3.64 & 26.96 & 2.75 & 1.00 & 1.36 \\
\hline $30 a$ & 0.0045 & 56.94 & 1.16 & 14.48 & 155.18 & 2.79 & 27.49 & 2.86 & 1.02 & 1.40 \\
\hline $30 b$ & 0.0043 & 49.14 & 1.16 & 7.87 & 58.30 & 2.17 & 21.71 & 2.86 & 1.00 & 1.34 \\
\hline $30 c$ & 0.0040 & 68.79 & 1.16 & 6.66 & 66.26 & 2.38 & 21.24 & 5.59 & 1.00 & 1.39 \\
\hline $60 a$ & 0.0046 & 814.26 & 1.16 & 12.61 & 87.15 & 2.40 & 26.26 & 3.60 & 1.00 & 1.34 \\
\hline $60 \mathrm{~b}$ & 0.0059 & 406.41 & 1.16 & 12.31 & 61.78 & 3.29 & 21.66 & 3.68 & 1.00 & 1.34 \\
\hline $60 c$ & 0.0035 & 60.41 & 1.16 & 8.57 & 75.75 & 2.68 & 29.12 & 3.72 & 1.00 & 1.33 \\
\hline $90 a$ & 0.0039 & 648.92 & 1.16 & 12.03 & 113.43 & 5.32 & 30.07 & 3.34 & 1.00 & 1.35 \\
\hline $90 \mathrm{~b}$ & 0.0044 & 239.91 & 1.16 & 11.28 & 69.53 & 2.24 & 26.02 & 3.91 & 1.00 & 1.39 \\
\hline $90 c$ & 0.0050 & 269.11 & 1.16 & 11.85 & 76.79 & 2.65 & 24.24 & 4.00 & 1.00 & 1.38 \\
\hline
\end{tabular}

Table 11. Image analysis results of hoop Specimen 3 (120-210 degrees).

\begin{tabular}{|c|c|c|c|c|c|c|c|c|c|c|}
\hline & \multirow{2}{*}{$\begin{array}{l}\text { Void } \\
\text { Volume } \\
\text { Fraction }\end{array}$} & \multicolumn{3}{|c|}{ Pore Area } & \multicolumn{3}{|c|}{$\begin{array}{l}\text { Nearest Neighbor } \\
\text { Distance }\end{array}$} & \multicolumn{3}{|c|}{ Aspect Ratio } \\
\hline & & $\begin{array}{l}\text { Max. } \\
(\mu \mathrm{m})\end{array}$ & $\begin{array}{l}\text { Min. } \\
(\mu \mathrm{m})\end{array}$ & $\begin{array}{l}\text { Avg. } \\
(\mu \mathrm{m})\end{array}$ & $\begin{array}{l}\text { Max. } \\
(\mu \mathrm{m})\end{array}$ & $\begin{array}{l}\text { Min. } \\
(\mu \mathrm{m})\end{array}$ & $\begin{array}{l}\text { Avg. } \\
(\mu \mathrm{m})\end{array}$ & $\begin{array}{l}\text { Max. } \\
(\mu \mathrm{m})\end{array}$ & $\begin{array}{l}\text { Min. } \\
(\mu \mathrm{m})\end{array}$ & $\begin{array}{l}\text { Avg. } \\
(\mu \mathrm{m})\end{array}$ \\
\hline $120 a$ & 0.0108 & 1560.59 & 1.16 & 24.73 & 74.90 & 4.33 & 23.69 & 3.34 & 1.00 & 1.32 \\
\hline $120 b$ & 0.0041 & 101.17 & 1.16 & 8.80 & 88.20 & 2.62 & 23.48 & 3.28 & 1.00 & 1.36 \\
\hline $120 c$ & 0.0034 & 92.21 & 1.16 & 7.03 & 58.45 & 2.80 & 24.19 & 3.27 & 1.00 & 1.32 \\
\hline $150 a$ & 0.0026 & 186.73 & 1.16 & 10.87 & 93.18 & 3.75 & 32.12 & 2.75 & 1.00 & 1.32 \\
\hline $150 b$ & 0.0043 & 75.73 & 1.16 & 8.41 & 98.30 & 3.08 & 22.42 & 9.33 & 1.00 & 1.40 \\
\hline $150 c$ & 0.0053 & 188.46 & 1.16 & 8.62 & 67.09 & 2.96 & 20.15 & 2.93 & 1.00 & 1.34 \\
\hline $180 a$ & 0.0026 & 108.11 & 1.16 & 9.22 & 89.88 & 2.17 & 29.39 & 5.00 & 1.00 & 1.50 \\
\hline $180 \mathrm{~b}$ & 0.0027 & 104.64 & 1.16 & 9.64 & 89.74 & 2.55 & 32.16 & 4.00 & 1.00 & 1.38 \\
\hline $180 \mathrm{c}$ & 0.0040 & 242.22 & 1.16 & 9.31 & 76.22 & 3.68 & 25.09 & 2.13 & 1.00 & 1.35 \\
\hline $210 a$ & 0.0034 & 297.43 & 1.16 & 8.15 & 110.74 & 2.55 & 22.75 & 2.53 & 1.00 & 1.33 \\
\hline $210 \mathrm{~b}$ & 0.0032 & 70.24 & 1.16 & 9.12 & 97.95 & 3.31 & 28.98 & 2.64 & 1.00 & 1.35 \\
\hline $210 \mathrm{c}$ & 0.0028 & 88.45 & 1.16 & 8.64 & 96.95 & 4.33 & 26.79 & 2.56 & 1.00 & 1.32 \\
\hline
\end{tabular}


Table 12. Image analysis results of hoop Specimen 3 (240-330 degrees).

\begin{tabular}{|c|c|c|c|c|c|c|c|c|c|c|}
\hline & \multirow{2}{*}{$\begin{array}{l}\text { Void } \\
\text { Volume } \\
\text { Fraction }\end{array}$} & \multicolumn{3}{|c|}{ Pore Area } & \multicolumn{3}{|c|}{$\begin{array}{l}\text { Nearest Neighbor } \\
\text { Distance }\end{array}$} & \multicolumn{3}{|c|}{ Aspect Ratio } \\
\hline & & $\begin{array}{l}\text { Max. } \\
(\mu \mathrm{m})\end{array}$ & $\begin{array}{l}\text { Min. } \\
(\mu \mathrm{m})\end{array}$ & $\begin{array}{l}\text { Avg. } \\
(\mu \mathrm{m})\end{array}$ & $\begin{array}{l}\text { Max. } \\
(\mu \mathrm{m})\end{array}$ & $\begin{array}{l}\text { Min. } \\
(\mu \mathrm{m})\end{array}$ & $\begin{array}{l}\text { Avg. } \\
(\mu \mathrm{m})\end{array}$ & $\begin{array}{l}\text { Max. } \\
(\mu \mathrm{m})\end{array}$ & $\begin{array}{l}\text { Min. } \\
(\mu \mathrm{m})\end{array}$ & $\begin{array}{l}\text { Avg. } \\
(\mu \mathrm{m})\end{array}$ \\
\hline $240 a$ & 0.0016 & 126.32 & 1.16 & 5.17 & 92.16 & 4.36 & 30.32 & 2.69 & 1.00 & 1.36 \\
\hline $240 b$ & 0.0022 & 147.42 & 1.16 & 6.06 & 96.24 & 2.43 & 26.94 & 3.58 & 1.00 & 1.40 \\
\hline $240 c$ & 0.0021 & 40.18 & 1.16 & 5.64 & 82.87 & 2.40 & 25.23 & 4.03 & 1.00 & 1.36 \\
\hline $270 a$ & 0.0025 & 118.80 & 1.16 & 7.54 & 88.59 & 2.31 & 30.65 & 2.93 & 1.00 & 1.32 \\
\hline $270 b$ & 0.0019 & 70.24 & 1.16 & 5.17 & 89.27 & 6.15 & 26.50 & 2.39 & 1.00 & 1.32 \\
\hline $270 c$ & 0.0022 & 53.76 & 1.16 & 5.02 & 80.94 & 2.19 & 23.72 & 7.22 & 1.00 & 1.43 \\
\hline $300 a$ & 0.0010 & 100.30 & 1.16 & 14.55 & 179.71 & 15.71 & 62.28 & 2.24 & 1.00 & 1.38 \\
\hline $300 \mathrm{~b}$ & 0.0027 & 322.87 & 1.16 & 10.91 & 153.68 & 6.53 & 33.79 & 4.15 & 1.00 & 1.49 \\
\hline $300 c$ & 0.0014 & 28.91 & 1.16 & 4.53 & 72.00 & 3.60 & 28.08 & 2.65 & 1.00 & 1.33 \\
\hline $330 a$ & 0.0009 & 18.79 & 1.16 & 4.76 & 101.92 & 1.89 & 29.10 & 2.61 & 1.00 & 1.36 \\
\hline $330 \mathrm{~b}$ & 0.0021 & 51.16 & 1.16 & 5.53 & 93.03 & 2.69 & 23.82 & 4.21 & 1.00 & 1.40 \\
\hline $330 \mathrm{c}$ & 0.0027 & 127.47 & 1.16 & 6.10 & 74.72 & 2.81 & 23.21 & 2.04 & 1.00 & 1.29 \\
\hline
\end{tabular}




\section{Summary}

ERDC's Geotechnical and Structures Laboratory conducted laboratory experiments to characterize the mechanical properties and microstructure characteristics of steel cylindrical shells. To obtain the tensile stress-strain material responses, uniaxial tensile experiments were performed on ASTM dog-bone specimens and hoop cross-sectional specimens at displacement rates of $0.1 \mathrm{in} . / \mathrm{min}$. In addition to the mechanical property tests, the microstructure of the steel hoop cross-sections specimens was analyzed using a commercially available image-analysis software program to determine the VVF, NND of pores, maximum and minimum pore sizes, and aspect ratio of the pores. The results of the investigation allow for an understanding of the mechanical behavior of steel cylindrical shells.

The relationships between microstructure and the mechanical properties of the steel cylindrical shells were analyzed to obtain parameters that will be used as part of a geostatistical techniques project to model the crosssectional porosity for mechanical analysis of crack initiation, growth, propagation, and coalescence when subjected to impulsive internal loads. 


\section{Reference}

Stone, T. Y., L. Tucker, Y. Hammi, T. N. Williams, H. El Kadiri, and M. F. Horstemeyer. 2009. Comparison of density measurement techniques for large PM components. Journal of Powder Metallurgy, Under Review. 


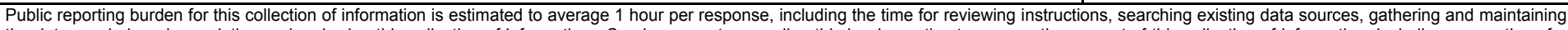

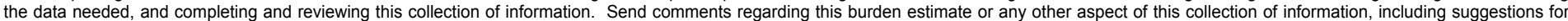

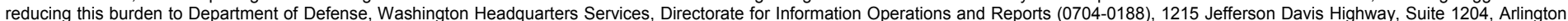

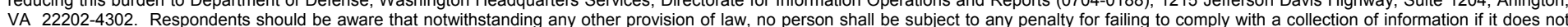
display a currently valid OMB control number. PLEASE DO NOT RETURN YOUR FORM TO THE ABOVE ADDRESS.

\begin{tabular}{|l|l|l}
\hline 1. REPORT DATE (DD-MM-YYYY) & 2. REPORT TYPE & 3. DATES COVERED (FrOm - To)
\end{tabular}

March 2012

Final

\section{TITLE AND SUBTITLE}

5a. CONTRACT NUMBER

Structure-Property Relationships of Steel Cylindrical Shells

5b. GRANT NUMBER

5c. PROGRAM ELEMENT NUMBER

6. AUTHOR(S)

5d. PROJECT NUMBER

Ruth G. Hidalgo-Hernandez, Paul G. Allison, Brett A. Williams,

Luis A. de Béjar, W. Scott Hart, and Jason Morson

5e. TASK NUMBER

5f. WORK UNIT NUMBER

7. PERFORMING ORGANIZATION NAME(S) AND ADDRESS(ES)

8. PERFORMING ORGANIZATION REPORT

NUMBER

Geotechnical and Structures Laboratory

U.S. Army Engineer Research and Development Center

ERDC/GSL TR 12-13

3909 Halls Ferry Road

Vicksburg, MS 39180-6199

9. SPONSORING / MONITORING AGENCY NAME(S) AND ADDRESS(ES)

U.S. Army Corps of Engineers

10. SPONSOR/MONITOR'S ACRONYM(S)

USACE

11. SPONSOR/MONITOR'S REPORT NUMBER(S)

\section{DISTRIBUTION / AVAILABILITY STATEMENT}

Approved for public release; distribution is unlimited.

\section{SUPPLEMENTARY NOTES}

\section{ABSTRACT}

Personnel of the U.S. Army Engineer Research and Development Center (ERDC), Geotechnical and Structures Laboratory (GSL), Engineering Systems and Materials Division (ESMD), Concrete and Materials Branch (CMB), Vicksburg, Mississippi, were asked to examine the mechanical properties and microstructure characteristics of a commercially available steel cylindrical shell in its asmanufactured condition. To examine the mechanical properties, uniaxial tensile experiments were performed on ASTM dog-bone specimens and hoop cross-sectional specimens at displacement rates of $0.1 \mathrm{in} . / \mathrm{min}$., to obtain the stress-strain material responses. A commercially available image-analysis program determined the void volume fraction (VVF), the nearest neighbor distance (NND) of pores, maximum and minimum pore sizes, and aspect ratio of the pores at 30-degree increments of the steel hoop cross-sections. The results of the investigation allow for an improved understanding of the mechanical behavior of steel cylindrical shells.

\section{SUBJECT TERMS}

Failure analysis

Structure properties

16. SECURITY CLASSIFICATION OF:

a. REPORT

Unclassified b. ABSTRACT

Unclassified
Cylindrical shells

Steel

c. THIS PAGE

Unclassified

Tensile experiments

17. LIMITATION OF ABSTRACT
18. NUMBER OF PAGES

20 19a. NAME OF RESPONSIBLE PERSON

19b. TELEPHONE NUMBER (include area code) 